\title{
Methamphetamine impairs social conformity during risk decision-making
}

DOI: $10.37716 /$ HBAB.2020010501

\author{
Hui $\mathrm{Ai}^{1, \dagger}$, Jiahui $\mathrm{Li}^{1, \dagger}$, Yuxuan $\mathrm{Zhang}^{1}$, Xue $\mathrm{Ma}^{1}$, Ti-fei Yuan ${ }^{2 *}$, Pengfei $\mathrm{Xu}^{3,4,5 *}$
}

Social conformity represents the propensity to align one's own attitude and behavior with the majority's, which is critical for adaptation to social norms. Methamphetamine abuse has been widely associated with risk-seeking and norm violation. However, whether risk attitude and behavior in individuals with methamphetamine use disorder (MUD) could be modulated by social conformity has remained unclear. Here, we report that MUD participants exhibited increased risk-taking behavior and reduced social conformity. Compared to controls, the MUD group showed decreased conformity when observing other's risk-averse decisions but increased conformity when observing other's risk-seeking decisions. Our model based on other-conferred utility (OCU) demonstrated attenuated OCU and increased risk preference in the MUD group. Importantly, OCU was significantly correlated to the degree of MA abuse. Taken together, our results suggest that social conformity is impaired in individuals with MUD, which might exacerbate their impulsivity in drug abuse behavior.

Keywords: decision-making, risk, social conformity, methamphetamine, other-conferred utility model

\section{INTRODUCTION}

Humans are naturally motivated to maintain meaningful social relationship with others (1). Social conformity implies the ability to incorporate others' responses into one's action and decisionmaking, which is beneficial to social integration (2). Whether to seek the reward of social acceptance or to avoid punishment for violation of social norms, conformity is underlain by reinforcing social feedback (3). Neuroimaging findings have shown a set of brain areas in response to conformity, including the ventromedial prefrontal cortex (vmPFC), anterior insula (AI), dorsal anterior cingulate cortex (dACC), and ventral striatum (2-5). While appropriate conformity could facilitate the elicitation of appropriate social responses and aid the obtainment of social approval (6), inappropriate conformity might result in maladaptive behavior or violation of social norms.

Abuse of illicit substances violates social norms and laws. Drug dependence, for example on methamphetamine (MA), is associated with impaired social functioning, increased aggression, as well as social isolation (7). These alterations are potentially due to disrupted frontal lobe functioning (8). In addition, individuals with methamphetamine use disorder (MUD) have a tendency to make risky decisions, accompanied by increased neural activity in the dorsolateral prefrontal cortex (dIPFC), AI, dACC, and striatum (911). Shared neural substrates for drug dependence and social conformity imply potential interactions between them. Although socialization and social conformity are crucial to initial drug abuse (12), the role of social conformity in risk preference of individuals with MUD remains unclear.

In the present study, MUD and healthy control (HC) participants were recruited for a social conformity task which required them to make decisions under different peer conditions with non-social (NS), social safe (SS), social risky (SR), and social mixed (SM) information (Fig. 1). Using a model that demarcates other-conferred

\footnotetext{
${ }^{1}$ Shenzhen Key Laboratory of Affective and Social Neuroscience, Magnetic Resonance Imaging Center, Center for Brain Disorders and Cognitive Sciences, Shenzhen University, 518060, Shenzhen, China. ${ }^{2}$ Shanghai Key Laboratory of Psychotic Disorders, Shanghai Mental Health Center, Shanghai Jiao Tong University School of Medicine, 200030, Shanghai, China. ${ }^{3}$ Beijing Key Laboratory of Applied Experimental Psychology, Faculty of Psychology, Beijing Normal University, 100875, Beijing, China. ${ }^{4}$ Center for Emotion and Brain, Shenzhen Institute of Neuroscience, 518057, Shenzhen, China. ${ }^{5}$ Guangdong-Hong Kong-Macao Greater Bay Area Research Institute for Neuroscience and Neurotechnologies, Hong Kong SAR, China.

$\dagger$ These authors contributed equally to this work.

*Corresponding authors. Email: ytf0707@126.com (T.Y.), pengfeixu.px@gmail.com (P.X.)
}

utility from subjective value (5), we aimed to evaluate the modulation of social conformity on risk preference and reward-seeking in individuals with MUD.

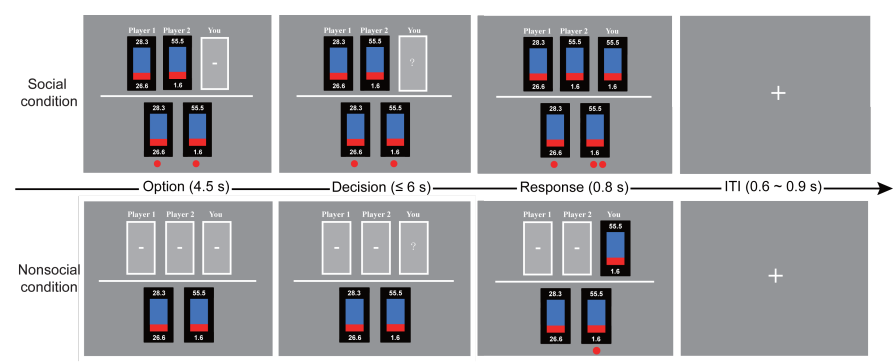

Fig. 1. Procedure of experimental task. In each trial, two options were presented for $4.5 \mathrm{~s}$ with (social) or without (nonsocial) information of two other players' decisions for reference. Participant had $6 \mathrm{~s}$ to make a selection from two options with the same probability to obtain different gains. The response/selection of the participant was presented for $0.8 \mathrm{~s}$ followed by a $0.6 \sim 0.9 \mathrm{~s}$ inter-trial interval (ITI).

\section{RESULTS}

Increased risk-taking behavior and reduced social conformity in MUD

In general, the MUD group showed significantly increased risktaking behavior compared to the HC group $(t(118)=3.221, p=$ 0.002 ; Fig. 2a). The conformity analysis by using a $2 \times 2$ ANOVA with Condition (SR and SS) as the within-participant factor and Group (MUD and HC) as the between-participant factor showed a significant main effect of Condition $(F(1)=11.829, p<0.001)$ and a significant Condition $\times$ Group interaction $(F(1)=7.967, p=$ 0.006), but a non-significant main effect of Group $(F(1)=0.409, p$ $=0.524$; Fig. 2b). Specifically, conformity was significantly higher in the SR compared to the SS condition $(p=0.048)$. Post hoc comparisons revealed that conformity was significantly higher in the SR condition compared to the SS condition in the MUD group $(F=37.950, p<0.001)$, but not in the HC group $(F=0.130, p=$ $0.721)$; conformity of the MUD group was significantly lower than that of the HC group in the SS condition $(F=5.570, p=0.020)$.

We investigated the relationship between social conformity and distance from others on the probability of choosing the risky option (see Materials and Methods on how distance was computed in the SR and SS conditions). Pearson's correlation analyses showed that conformity correlated significantly with distance in the SS condition 
( $r=-0.791, p<0.001)$ but not the SR condition $(r=-0.193, p=$ $0.299)$ in the HC group. Conformity correlated significantly with distance in both SR $(r=-0.701, p<0.001)$ and SS conditions $(r$ $=-0.633, p<0.001)$ in the MUD group (Fig. 2c). The correlation coefficient was significantly higher in the SR condition compared to the SS condition in the HC group $(Z=-3.059, p=0.002)$ but not in the MUD group $(Z=-0.826, p=0.409)$. For the SR condition, there was a significant group difference between correlation coefficients, with the MUD showing a stronger negative association between social conformity and distance compared to the HC group $(Z=$ $3.097, p<0.001)$. For the SS condition, there was only a marginally significant group difference between correlation coefficients $(Z=$ $1.506, p=0.066)$.
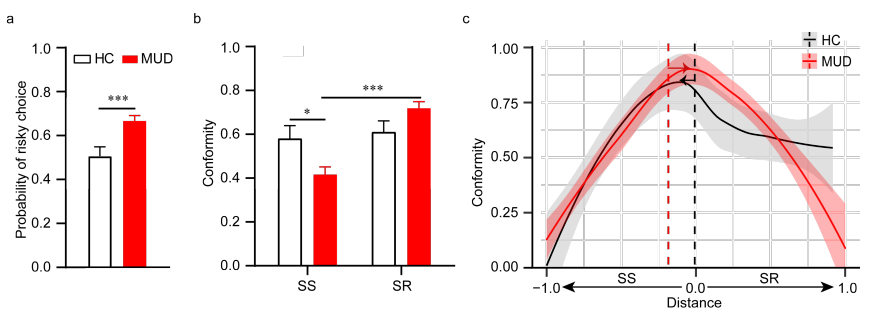

Fig. 2. Risk behavior and social conformity. (a) Probability of risky choice in the two groups; (b) Social conformity of the two groups in SS and SR conditions; (c) Social conformity as a function of social distance between self and others in SS and SR conditions. NS, nonsocial condition; SM, social mixed condition; SS, social safe condition; SR, social risky condition; $\mathrm{HC}$, healthy control; MUD, methamphetamine use disorder

Next, we conducted a mixed effect logistic regression model to examine the modulation of social conformity on sensitivity toward reward probability in the MUD group, with likelihood of risky choice as the dependent variable and Group (MUD and HC), Condition (NS, SS, and SR), and Probability for a high potential gain $(40 \%, 50 \%, 60 \%, 70 \%, 80 \%$, and $90 \%)$ as independent variables. The results of the mixed effect model revealed that Condition $(Z=2.908, p=0.004)$, Group $(Z=-3.454, p<0.001)$, interaction between Probability and Condition $(Z=-1.982, p<$ $0.048)$ significantly contributed to the likelihood of risky choice (Fig. S2). See Tables S1-5 for results of $F$-tests and post hoc tests with Tukey's corrections for multiple comparisons.

\section{Model analyses}

In combination of the power function of prospect theory and the OCU model (5), we estimated preferences of risk and social conformity simultaneously (see Materials and Methods for model specifications).
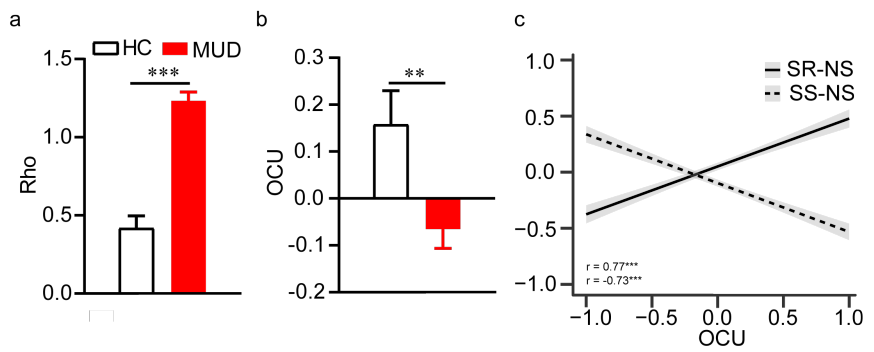

Fig. 3. Risk and social conformity preferences. (a) Estimated risk preference (Rho) in the groups; (b) Estimated social conformity preference in the two groups; (c) Social conformity preference was highly correlated with the difference between probability of risky choice in social risky and nonsocial conditions (SR-NS), as well as with that between probability of risky choice in social safe and nonsocial conditions (SS-NS) across the two groups.
The independent-sample $t$-test on risk preference (Rho) showed a significant difference between the two groups $(t(113)=7.798, p<$ 0.001 ; Fig. 3a). The independent-sample $t$-test on other-conferred utility (OCU) also showed a significant group difference $(t(113)$ $=2.71, p=0.008$; Fig. 3b). There was a significantly positive correlation of OCU with the difference of probability on risky option selection between SR and NS $(r=0.769, p<0.001)$ and a significantly negative correlation with the difference between SS and NS ( $r=-0.734, p<0.001$; Fig. 3c) across the two groups, confirming that the OCU model could differentially predict social influences on individual's choices in different situations.

\section{Social conformity abnormality in MUD}

Partial correlation analyses revealed that after controlling for age, age at first MA use and level of education, OCU was negatively correlated with amount of MA use ( $\Delta$ amount, $r=-0.232, p=0.034$, Fig. 4a), but not frequency of MA use ( $\Delta$ frequency, $r=0.200, p=$ 0.068), Fig. 4b).
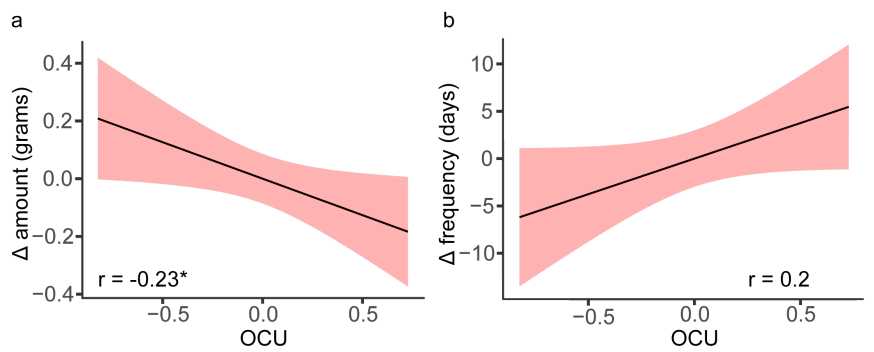

Fig. 4. Severity of methamphetamine (MA) use contributed to deficit of social conformity. Social conformity preference as a function of (a) amount and (b) frequency of MA intake. Note that $\Delta$ indicates results of partial correlation analyses that controlling for age, age at first MA use and educational level.

\section{DISCUSSION}

The present study reported a model-based explanation of reduced social conformity during risk decision-making in individuals with MUD. The results indicated the "rigid" type of risk decision-making in individuals with MUD. The effect is correlated to their drug intake amount, indicating a potential link between reduced social conformity and addictive behavior.

Social influences on drug intake behavior are vast and bidirectional (7). Adolescents are prone to drug intake or impulsive behavior, with peer pressure acting as a high-risk factor for progression into abuse (13). While social support and society re-entry programs are effective for relapse prevention and full rehabilitation from addiction (14), social violation is a common behavior for drug dependents (15). Social conformity therefore represents a potential evaluation index for social functioning impairment and/or an effectiveness indicator for rehabilitation programs. The OCU model provides an integrated function which incorporates others' choices into one's own risk-taking behavior (5). Our current crosssectional analyses, however, did not fully address the possibility that social conformity changes during abstinence; this warrants future longitudinal design to elucidate the trajectory of social conformity functioning at different stages of drug addiction.

Social signals of danger confer the boundary of "safe" behavior. The reduced social conformity during risk decision-making therefore contributes to the increased risky behavior. Previous studies reported higher impulsivity in individuals with MUD, correlated to reduced D2 receptor availability at the striatum and enhanced mesocorticolimbic functional connectivity (11). The aberrant neural 
connectivity and molecular signaling in ventromedial prefrontal cortex and ventral striatum might contribute the altered coding and functioning of social conformity signals $(9,10)$. This yet requires further neuroimaging studies on social conformity in individuals with MUD.

Consistent with previous studies of social conformity, we observed an asymmetric pattern in the way that others' choices could influence observers' choices. In general, people conformed more with others' safe option selection than others' risky option selection. However, this asymmetry was absent in the MUD group, who showed high conformity with others when observing risktaking behavior. These results suggest that individuals with MUD are not only risk-seeking themselves, but also susceptible to others' risky behavior. Therefore, from a social perspective, successful addiction intervention might well depend on the avoidance of social influence and peer pressure in order to prevent or reduce drug intake behavior in individuals with MUD. It has been proposed that conformity behavior is driven by informational and normative motivations which aim to facilitate appropriate social responses and promote social acceptance respectively $(6,16)$. Although informational motivation was not experimentally differentiated from normative motivation in the present study, the increased risk preference together with decreased social conformity jointly indicate excessive reward-seeking regardless of informative and normative suggestions in individuals with MUD.

This study has some limitations. Firstly, the sample size was relatively small, and limited to participants during a period abstinence from MA; it would be interesting to recruit active drug users with MUD and measure their social conformity level. Generalizability of the findings might also have been compromised by exclusion of heroin-mixed use participants, which is common in individuals with MUD (17). Secondly, the study did not address any potential difference between social signals from "drug use partners" and those from "anonymous partners". Earlier findings suggested that social signals could transmit information about relapse and/or increase drug abuse behavior (18). Investigation into the degree of social conformity to "drug use partners" in drug intake behavior would provide clinically useful information in relapse risk evaluation. Thirdly, although the neural substrates underlying social conformity have previously been examined, the current study did not include any neurophysiological measurements during the task; it would be helpful to identify potential neural processing differences during social conformity decision-making between healthy individuals and those with MUD, which would in turn provide potential targets for future intervention with brain stimulation, for instance.

In conclusion, our results demonstrated reduced social conformity of individuals with MUD in risk decision-making. The findings refine our understanding on how social signals influence decision-making in MUD individuals. The results also support the wide implementation of social-based addiction intervention.

\section{MATERIALS AND METHODS}

\section{Participants}

A total of 89 male individuals with MUD and 31 healthy control (HC) participants were recruited for this study (Table 1). The inclusion criteria of MUD were as follows: 1) met the Diagnostic and Statistical Manual of Mental Disorders, 5th Edition (DSM-5) criteria in a structured interview; 2) had been using only MA (and no other drug) for at least one year; 3) the use of MA lasted for at least a month with frequency of intake of at least three times per week; and 4) the amount of MA intake was more than $0.1 \mathrm{~g}$ per day prior to abstinence. All participants had normal or correctedto-normal vision. None of the participants reported any current neurological diseases, or other severe bodily diseases. The study was approved by ethic committee of Shenzhen University, and all procedures were in accordance with the Declaration of Helsinki. All participants provided written informed consent and participated in the study voluntarily.

\begin{tabular}{|c|c|c|c|c|}
\hline & $\underset{(n=31)}{\text { HC }}$ & $\begin{array}{c}\text { MUD } \\
(n=89)\end{array}$ & $t$ & $p$ \\
\hline Age (years) & $33.87 \pm 7.637$ & $33.17 \pm 6.042$ & -0.453 & 0.653 \\
\hline Education (level ${ }^{*}$ ) & $2.17 \pm 0.592$ & $2.00 \pm 0.512$ & -1.471 & 0.144 \\
\hline Duration of MA use before abstinence (years) & NA & $6.8 \pm 3.484$ & NA & NA \\
\hline Duration of abstinence from MA use (days) & NA & $263.27 \pm 258.351$ & NA & NA \\
\hline Age at first MA use (years) & $\mathrm{NA}$ & $25.43 \pm 6.828$ & NA & NA \\
\hline Amount of MA (grams per day) & NA & $0.539 \pm 0.4105$ & NA & NA \\
\hline Frequency of MA use (days) & NA & $7.74 \pm 14.184$ & NA & NA \\
\hline BIS & $58.33 \pm 10.036$ & $61.39 \pm 8.772$ & 1.596 & 0.114 \\
\hline
\end{tabular}

\section{Task design}

For the task, information on risk and social conformity was presented as previously described (5). In each trial, participants made choices between two options (Fig. 1). Each option consists of two probabilities to obtain high and low potential gains, respectively. The two options in each trial have same probabilities but different magnitudes of gains. The option with a smaller variance of magnitude was defined as the safe option, while the one with a larger variance of magnitude was the risky option. Participants made decisions either with or without the knowledge of the other two players' selection. Participants were instructed that their choices could also be randomly selected to provide to others anonymously in the same way. At the end of the experiment, participants were paid based on their responses to one randomly selected trial during the experiment.

There were four conditions in the manipulations of social information, including 1) nonsocial condition (NS) in which no information about others' decisions was provided, 2) social safe condition (SS) in which information about the selection of the safe option by both of the other players was provided, 3) social risky condition (SR) in which information about the selection of the risky option by both of the other players was provided, and 4) social mixed condition (SM) in which information on the different choices selected by the other two players (i.e., one selected the safe while the other selected the risky option) was provided. For each condition, there were six levels of probability $(40 \%, 50 \%$, $60 \%, 70 \%, 80 \%$, and $90 \%$ ) to obtain a high potential gain. Pairs of high and low potential gain were selected from a previous study (5). In total, the experiment consisted of 96 trials with 24 per condition and 4 per level of probability. All trials were randomly presented across participants using PsychToolbox-3 in MATLAB 2018a (MathWorks).

\section{Data analyses}

In general, to examine social influence on risk-taking behavior in the MUD group, we analyzed behavioral responses to task manipulation (absence and presence of social information) and psychological preferences of risk and social conformity with the 
proposed model that in combination of other-conferred utility with power utility function (5). Specifically, we calculated probabilities of choosing the risky option and social conformity across NS, SS, SR, and SM conditions in MUD and HC groups. Social conformity was defined in terms of the probability of selecting the same option as other two players in the SS and SR conditions. To test the modulation of social influence on the sensitivity towards reward probability, we calculated the probability of choosing the risky option across different levels of probability of obtaining a high potential gain in the two groups. To test the relationship between social conformity and distance from others on the probability of choosing the risky option, we defined distance in SR as $(0-p)$ and distance in SS as $(1-p)$, where $p$ was the probability of choosing the risky option in NS.

\section{Subjective preference estimation}

In the current study, we focused on subjective valuation of risk and social conformity. Integrating other-conferred utility (OCU) with power utility function, a recent proposed model has been shown to fit individual's decisions better than models of making decision fully following others or not at all, or changing risk preference from absence to presence of information about others' choices (5). On the basis of the OCU model, we estimated risk preference and social conformity preference in the two groups.

$$
\begin{gathered}
u(\text { nonsocial })=P_{\text {high }}\left(V_{\text {high }}\right)^{\rho}+\left(1-P_{\text {high }}\right)\left(V_{\text {low }}\right)^{\rho} \\
u(\text { social })=u(\text { nonsocial })+\text { ocu } \\
P(\text { riskyoptionselection })=(1+\exp (-\mu(u(\text { risky })-u(\text { safe }))\})^{-1}
\end{gathered}
$$

Where, $u$ (nonsocial) represent the utility of nonsocial option, either risky or safe. $P_{\text {high }}$ and $V_{\text {high }}$ indicate the probability and value of a high potential gain in each option respectively. $\mu$ is sensitivity to changes of subjective difference between the two options. The curvature of the utility function $\rho$ represents risk preference, which indicating risk-averse when $\rho<1$ and risk-seeking when $\rho>1$. ocu indicates OCU which was only added to the utility of SR and $\mathrm{SS}$, but not SM or NS. Because the sign and range of OCU were not restricted in the estimation, OCU was scaled to the range between -1 and 1 using the following equation.

$$
z(o c u)=2 \times\left(\frac{o c u_{i}-\min (o c u)}{\max (o c u)-\min (x o c u)}\right)-1
$$

\section{Statistical analyses}

An independent-sample $t$-test was conducted to examine risktaking behavior in the MUD group. Associations of conformity with risk preference distance from others were tested with Pearson's correlation analyses. To examine the modulation of social conformity on sensitivity toward reward probability in the MUD group, we conducted a mixed effect logistic regression model using the lme4::1mer in R version 3.5.2 (http://www.r-project.org/). To examine the contribution of MA use in social conformity preference of individuals with MUD, we conducted partial correlation analyses to test the relationships of OCU with amount and frequency of MA use in the MUD group, and with age, age at first MA use, and educational level as covariates.

\section{SUPPLEMENTARY MATERIALS}

Supplementary materials for this article are available at http://www.humanbab.net/qfy-content/ uploads/2020/10/2020010501SM.pdf

Supplementary Table 1. Results of the mixed effect model with Choice as the dependent variable and with Probability, Condition, Group, and Magnitude of Potential Gain as independent variables.

Supplementary Table 2. Simple comparisons for the interaction between Probability and Condition. The $p$ value was adjusted using Tukey's method.

Supplementary Table 3. Simple comparisons for the main effect of Probability. The $p$ value was adjusted using Tukey's method.

Supplementary Table 4. Simple comparisons for the main effect of Condition. The $p$ value was adjusted using Tukey's method.

Supplementary Table 5. Simple comparison for the main effect of Group. The $p$ value was adjusted using Tukey's method.

Supplementary Fig. 1. Probability of risky choice in four conditions across groups. NS, nonsocial condition; SM, social mixed condition; SS, social safe condition; SR, social risky condition $\mathrm{HC}$, healthy control; MUD, methamphetamine use disorder.

Supplementary Fig. 2. Probability of risky choice increased with increased probability of high potential gain, which interacted with Condition and Group. NS, nonsocial condition; SS, social safe condition; SR, social risky condition; $\mathrm{HC}$, healthy control; MUD, methamphetamine use disorder.

\section{REFERENCES}

1. J. K. Rilling, A. G. Sanfey, The neuroscience of social decision-making. Annu. Rev. Psychol. 62 , 23-48 (2011).

2. R. B. Cialdini, N. J. Goldstein, Social influence: Compliance and conformity. Annu. Rev. Psychol. 55, 591-621 (2004).

3. V. Klucharev, K. Hytönen, M. Rijpkema, A. Smidts, G. Fernández, Reinforcement learning signal predicts social conformity. Neuron 61, 140-151 (2009).

4. M. Edelson, T. Sharot, R. J. Dolan, Y. Dudai, Following the crowd: Brain substrates of long-term memory conformity. Science 333, 108-111 (2011).

5. D. Chung, G. I. Christopoulos, B. King-Casas, S. B. Ball, P. H. Chiu, Social signals of safety and risk confer utility and have asymmetric effects on observers' choices. Nat. Neurosci. 18, 912-916 (2015)

6. U. Toelch, R. J. Dolan, Informational and normative influences in conformity from a neurocomputational perspective. Trends Cogn. Sci. 19, 579-589 (2015).

7. B. D. Homer, T. M. Solomon, R. W. Moeller, A. Mascia, L. DeRaleau, P. N. Halkitis, Methamphetamine abuse and impairment of social functioning: A review of the underlying neurophysiological causes and behavioral implications. Psychol. Bull. 134, 301-310 (2008).

8. R. Z. Goldstein, N. D. Volkow, Dysfunction of the prefrontal cortex in addiction: Neuroimaging findings and clinical implications. Nat. Rev. Neurosci. 12, 652-669 (2011).

9. M. Kohno, A. M. Morales, D. G. Ghahremani, G. Hellemann, E. D. London, Risky decision making, prefrontal cortex, and mesocorticolimbic functional connectivity in methamphetamine dependence. JAMA Psychiatry 71, 812-820 (2014).

10. M. P. Paulus, S. F. Tapert, M. A. Schuckit, Neural activation patterns of methamphetamine dependent subjects during decision making predict relapse. Arch. Gen. Psychiatry 62, 761 768 (2005).

11. M. Kohno, K. Okita, A. M. Morales, C. L. Robertson, A. C. Dean, D. G. Ghahremani, F. W. Sabb, R. A. Rawson, M. A. Mandelkern, R. M. Bilder, E. D. London, Midbrain functional connectivity and ventral striatal dopamine D2-type receptors: Link to impulsivity in methamphetamine users. Mol. Psychiatry 21, 1554-1560 (2016).

12. R. L. Gorsuch, M. C. Butler, Initial drug abuse: A review of predisposing social psychological factors. Psychol. Bull. 83, 120-137 (1976).

13. T. H. Brandon, T. A. Herzog J. E. Irvin, C. J. Gwaltney Cognitive and social learning models of drug dependence: Implications for the assessment of tobacco dependence in adolescents. Addiction 99, 51-77 (2004).

14. P. L. Dobkin, M. D. Civita, A. Paraherakis, K. Gill, The role of functional social support in treatment retention and outcomes among outpatient adult substance abusers. Addiction 97, 347-356 (2002).

15. G. Chen, Gender differences in crime, drug addiction, abstinence, personality characteristics, and negative emotions. J. Psychoactive Drugs 41, 255-266 (2009).

16. M. Deutsch, H. B. Gerard, A study of normative and informational social influences upon individual judgment. J. Abnorm. Psychol. 51, 629-636 (1955).

17. M. C. Meacham, S. A. Strathdee, G. Rangel, R. F. Armenta, T. L. Gaines, R. S. Garfein, Prevalence and correlates of heroin-methamphetamine co-injection among persons who inject drugs. $J$. Stud. Alcohol Drugs 77, 774-781 (2016).

18. R. Niaura, Cognitive social learning and related perspectives on drug craving. Addiction 95 S155-163 (2000).

Acknowledgments: The authors thank the lab members for their support. Funding: This work was supported by the National Natural Science Foundation of China (31871137, 31700959, 31920103009, 31530031, and 31671133), Young Elite Scientists Sponsorship Program by China Association for Science and Technology (2018), Guangdong Key Basic Research Grant (2018B030332001), Guangdong young Innovative Talent Project (2016KQNCX149), Guangdong Pearl River Talents Plan Innovative and Entrepreneurial Team Grant (2016ZT06S220), Shenzhen Science and Technology Research Funding Program (JCYJ20180507183500566 and CYJ20170412164413575), and Shenzhen Peacock Program (827000235, KQTD2015033016104926). Author contributions: TFY and PX designed the study; HA, $\mathrm{JL}, \mathrm{YZ}$ and $\mathrm{XM}$ conducted the study; JL, TFY and PX analyzed the data; HA, TFY and PX wrote the manuscript; all authors have read and approved the final version of the manuscript. Competing interests: The authors declare no competing financial interests. Data and materials availability: All data and materials are available are upon request to the corresponding authors. 